\title{
A Correlative Study of Hemodynamically Significant Patent Ductus Arteriosus and Platelets within 24 hours of Birth in Preterm Infants
}

\author{
Yi Ren \\ xuzhou hospital affiliated to southeast university \\ Xiangyu Gao ( $\nabla$ ggaoxyy@163.com ) \\ Xuzhou University affiliated to southeast unversity \\ Huiying Wang \\ xuzhou hospital affliated to southeast university \\ Bo Yang \\ Xuzhou Hospital Affiliated to Southeast University \\ Bao Jin \\ xuzhou hospital affiliated to southeast university \\ Honglin Lei \\ xuzhou hospital affiliated to southeast university \\ Dandan Zhao \\ xuzhou hospital affiliated to southeast university \\ Di Huang \\ xuzhou hospital affiliated to southeast university
}

Research article

Keywords: platelet count; plateletocrit; patent ductus arteriosus; infant; preterm

Posted Date: August 31st, 2019

DOI: https://doi.org/10.21203/rs.2.13804/v1

License: @ (i) This work is licensed under a Creative Commons Attribution 4.0 International License. Read Full License 


\section{Abstract}

Objective To investigate the correlation between the hemodynamically significant patent ductus arteriosus (hsPDA) of preterm infants and the platelet count (PLT), plateletocrit within $24 \mathrm{~h}$ of birth.Methods The clinical data of 1270 preterm infants were retrospectively studied. The patients were divided into the nonPDA group, no hsPDA group, and hsPDA group. Results The smaller gestational age and the lower birth weight, PLT, plateletocrit were associated with the greater likelihood of PDA $(\mathrm{P}<0.05)$. The receiver operating characteristic $(\mathrm{ROC})$ curve showed that the predictive values of PLT and plateletocrit were 0.703 and 0.748 , respectively, with the best critical values of $241.5 \times 109 / \mathrm{L}$ and $0.245 \%$. For the preterm infants with PLT $<241.5 \times 109 / \mathrm{L},<150 \times 109 / \mathrm{L}$ and $<$ $100 \times 109 / \mathrm{L}$ as well as plateletocrit $<0.245 \%$ and $0.09 \%$, the risks of hsPDA were $1.876,2.169,6.216,1.749$ and 5.407 times of preterm infants with PLT $\geq$ $241.5 \times 109 / \mathrm{L}, \geq 150 \times 109 / \mathrm{L}$ and $\geq 100 \times 109 / \mathrm{L}$ as well as plateletocrit $\geq 0.245 \%$ and $\geq 0.09 \%$. The logistic regression analysis showed that plateletocrit was an independent risk factor for preterm infants with hsPDA $(P=0.000)$.Conclusions The decrease in plateletocrit of the preterm infants within $24 \mathrm{~h}$ of birth was the independent risk factor of hsPDA on the 4th-7th day of birth.

\section{Background}

The arterial duct connecting the root of the aorta and left pulmonary artery near the pulmonary artery bifurcation is an important channel for fetal circulation. The arterial duct of the vast majority of term infants will be closed immediately within 72 hours after birth. For the preterm infants, the arterial duct often cannot be closed due to various issues such as arterial duct wall dysplasia and abnormal secretion of prostaglandins. The left-to-right shunt would be caused, leading to an increase in the pulmonary circulation blood volume and left ventricular volume overload as well as a decrease in systemic circulation blood volume. Further, as the pulmonary artery smooth muscle development of preterm infants is imperfect, the decrease in pulmonary vascular resistance is more significant, the shunt volume is larger, and the pulmonary edema, pulmonary injury, bronchopulmonary dysplasia, heart failure and necrotizing enterocolitis would more easily occur $[1,2]$. Therefore, it is extremely important to understand the arterial duct closure influencing factors and development mechanism for the active prevention and proper intervention of patent ductus arteriosus (PDA) on the preterm infants.

The recent studies found that the platelets of ductus arteriosus might play an important role in the arterial duct closure process and the platelet adhesion and aggregation were critical steps for the closure of ductus arteriosus. The thrombocytopenia not only was an independent predictive factor of preterm infants with ductus arteriosus closure failure [3] but also significantly increases the risk of hemodynamically significant patent ductus arteriosus (hsPDA) [4]. However, there is still considerable controversy about the role of platelets in preterm infants with hsPDA. In order to understand the relationship between the platelets and hsPDA of preterm infants, here we conducted a retrospective cohort study.

\section{Methods}

\section{Patients}

The present study was approved by the Ethics Committee of Children's Medical Center of Xuzhou Hospital affiliated to Southeast University, China. Written informed consent was obtained from all participants.

A total of 2594 preterm infants admitted into Department of Neonatology, the Affiliated Xuzhou Hospital of Southeast University from March 2013 to February 2019 were enrolled. The clinical data were retrospectively analyzed. According to the inclusion criteria, 1457 cases were selected. Further, 187 cases were excluded due to the exclusion criteria, and 1270 cases were finally included.

Inclusion criteria: infants whose gestational age < 34 weeks; infants admitted into our department within $24 \mathrm{~h}$ after birth, followed by the venous blood routine examination; infants receiving echocardiography examination on $4^{\text {th }}-7^{\text {th }}$ day after birth.

Exclusion criteria: infants with un-trusted or incomplete medical history data and hospitalization data.

\section{Diagnosis criteria for hemodynamically significant patent ductus arteriosus (hsPDA)}

Based on the criteria ([5] ) quoted in Avery's Diseases of the Newborn (9th ed) [1]: patients who meet three out of six items of the following clinical manifestations and all three items of echocardiographic diagnosis criteria were diagnosed with hsPDA. The six clinical manifestations include hearable systolic or continuous murmur on the left sternal border, increased precordial pulse, Corrigan's pulse, resting heart rate more than 180 beats/min, unexplained breathing deterioration, and increased pulmonary vascular shadow, enlarged heart or signs of pulmonary edema shown by the chest radiography. The three echocardiographic diagnosis criteria include left atrial internal diameter/aortic internal diameter over 1.4, regurgitated pulmonary valve during the diastolic period, and PDA duct internal diameter more than $1.5 \mathrm{~mm}$.

\section{Diagnosis criteria for non-hemodynamically significant patent ductus arteriosus (nhsPDA)}

The preterm infants were diagnosed as PDA by the echocardiography on the $4^{\text {th }}-7^{\text {th }}$ day after birth, without achieving the above-mentioned hsPDA diagnosis criteria. 
The closure of arterial duct of preterm infant was confirmed by the echocardiography on the $4^{\text {th }}-7^{\text {th }}$ day after birth.

\section{Diagnosis criteria for thrombocytopenia}

The definition of the thrombocytopenia in the peripheral venous blood of the newborns (including preterm infants) is relatively uniform, that is PLT < $150 \times 10^{9} / \mathrm{L}[6]$. Mild reduction: $100 \times 10^{9} / \mathrm{L} \leq \mathrm{PLT}<150 \times 10^{9} / \mathrm{L} ;$ moderate reduction: $50 \times 10^{9} / \mathrm{L} \leq \mathrm{PLT}<100 \times 10^{9} / \mathrm{L} ;$ severe reduction: PLT < $50 \times 10^{9} / \mathrm{L}[6$, 7]. The reference values of other parameters of the platelets: plateletocrit: $0.09-0.3 \%$, platelet distribution width (PDW): $14-18 \%$, mean platelet volume (MPV): 7.0-11.0 fL, and platelet-large cell ratio (P-LCR): 12-35\%.

\section{Study indicators}

The multi-center large sample data (47,291 newborns, with the gestational age of 22 to 42 weeks) showed that PLT was increased slightly with the increase in the gestational age and day-age [8]. Therefore, the data of blood platelet parameters (PLT, PCT, PDW, MPV, P-LCR) of the preterm infants within $24 \mathrm{~h}$ of birth were retrospectively analyzed. Besides, the clinical factors possibly related to the occurrence of PDA were also recorded, including gender, early birth, gestational age, gestational hypertension, hormone course from $24 \mathrm{~h}$ to $7 \mathrm{~d}$ ahead of delivery, preterm rupture of membranes for greater than $18 \mathrm{~h}$, cesarean delivery, birth weight, and premature birth [1, 2, 9]. Further, we recorded whether 5-min Apgar score was less than 7 points, whether there was respiratory distress syndrome, whether the days under positive pressure ventilation were more than 3 days, as well as the fluid intake volume and urine output on the $1^{\text {st }}$ $3^{\text {rd }}$ day after birth. Finally, the echocardiography was performed on the $4^{\text {th }}-7^{\text {th }}$ day (United States GE LOGIQ-E9 type chronoscope ultrasonic diagnostic equipment), and the patients were divided into the nPDA group (982 cases, 77.3\%), nhsPDA group (178 cases, 14.0\%), and hsPDA group (110 cases, 8.7\%).

\section{Statistical Analysis:}

Statistical Analysis was conducted using the SPSS 20.0 software. The count data were shown as cases (\%) and compared with Likelihood Ratio Chi-square Test of line $\times$ list data, and the pairwise comparison was carried out with chi-square test of fourfold table data (Pearson chi-square test/continuous correction chi-square test/Fisher's exact test). The measurement data normally distributed in the three groups were expressed as mean \pm standard deviation and the means among the groups were compared with One-way ANOVA LSD method or Tamhane's T2 method for independent samples. The comparisons of the predictive values for predicting the hsPDA of preterm infants by relevant factors were carried out by receiver operating characteristic (ROC) curve. Multi-factor correlation analysis was performed using binary Logistic regression analysis (forward biased maximum likelihood estimation method). $P<0.05$ showed that the difference was statistically significant.

\section{Results}

\section{Demographic characteristics grouped according to the preterm infants' artery ducts}

There was no statistically significant difference in the early birth cases, gestational age, gestational hypertension cases, full hormone course cases, preterm rupture of membrane cases, cesarean delivery cases, birth weight, and premature birth cases between the three groups $(P>0.05)$. Besides, the difference was not significant in the case number of 5-min Apgar score less than 7 points, respiratory distress syndrome, and positive pressure ventilation more than 3 days, as well as the fluid intake volume and urine output on the $1^{\text {st }}-3^{\text {rd }}$ day after birth, platelet distribution width (PDW), mean platelet volume (MPV), and plateletlarge cell ratio (P-LCR) within $24 \mathrm{~h}$ after birth $(P>0.05)$.

The differences in the gestational age, birth weight, PLT, and plateletocrit were statistically significant $(P=0.035,0.000,0.000$, and 0.000 , respectively). The pairwise comparison showed that the difference in the gestational age among the three groups was not statistically significant $(P>0.05)$. For birth weight, the differences between the nPDA group and hsPDA group and between the nhsPDA group and hsPDA group were statistically significant $(P=0.000$ and 0.019 , respectively). Moreover, there were significant differences in the platelet counts (PLT) and plateletocrit between groups $(P<0.05)$. Importantly, the smaller gestational age and the lower birth weight, PLT, plateletocrit levels within $24 \mathrm{~h}$ after birth were associated with the greater likelihood of nhsPDA or hsPDA (Table 1).

\section{Predictive values for predicting hsPDA of the preterm infants by PLT and plateletocrit within $24 \mathrm{~h}$ after birth}

The areas under the ROC curve for predicting the hsPDA of preterm infants by the gestational age and birth weight were 0.528 and 0.578 , respectively, and the predictive value was lower. The areas under the ROC curve for predicting the hsPDA of preterm infants by PLT and plateletocrit within $24 \mathrm{~h}$ after birth were $0.703(95 \% \mathrm{Cl} 0.655-0.751, P=0.000)$ and $0.748(95 \% \mathrm{Cl} 0.699-0.797, P=0.000)$, respectively, and the predictive value was moderate. The best predicting critical values of the PLT and plateletocrit were $241.5 \times 10^{9} / \mathrm{L}$ (sensitivity, $78.2 \%$; specificity, $67.0 \%$ ) and $0.245 \%$ (sensitivity, $68.2 \%$; specificity, $74.2 \%$ ), respectively (Figure 1 and Table 2). 


\section{Single factor correlation analysis of PLT and hematocrit within $24 \mathrm{~h}$ after birth with the hsPDA of the preterm infants}

For the preterm infants with PLT $<241.5 \times 10^{9} / \mathrm{L},<150 \times 10^{9} / \mathrm{L}$, and $<100 \times 10^{9} / \mathrm{L}$ as well as plateletocrit $<0.245 \%$ and $<0.09 \%$ within $24 \mathrm{~h}$ after birth, the risks of hsPDA were $1.876,2.169,6.216,1.749$, and 5.407 times of the preterm infants with PLT $\geq 241.5 \times 10^{9} / \mathrm{L}, \geq 150 \times 10^{9} / \mathrm{L}$, and $\geq 100 \times 10^{9} / \mathrm{L}$ as well as plateletocrit $\geq 0.245 \%$ and $\geq 0.09 \%$, respectively (Table 3 ).

\section{Multiple factor correlation analysis of the hsPDA on the preterm infants}

The binary logistic regression analysis (partial maximum likelihood estimation forward method) was applied, and $P \leq 0.05$ and $P>0.1$ were taken as the variable inclusion and exclusion criteria, respectively. The results showed that the gestational age and PLT had no independent correlation with the hsPDA of preterm infants $(P=0.911$ and 0.392 , respectively); therefore, the two independent variables were excluded. Birth weight and plateletocrit were independent risk factors for the hsPDA of preterm infants, with the $P$ values of 0.000 and 0.000 , respectively. By the further classification assignment, the results showed that the risk of hsPDA would be increased by 1.303 times $(95 \% \mathrm{Cl} 1.052-1.613)$ if the birth weight of the preterm infants was reduced by $500 \mathrm{~g}$; the risk of hsPDA would be increased by 3.328 folds $(95 \% \mathrm{Cl} 2.455-4.512)$ if the plateletocrit was reduced by $0.10 \%$ (Table 4$)$.

\section{Discussion}

In this study, we explored the role of platelets in preterm infants with hsPDA and found that the smaller gestational age and the lower birth weight, PLT, plateletocrit were associated with the greater likelihood of PDA. The ROC curve and single factor correlation analysis showed that PLT and plateletocrit were the predictive factors of hsPDA. The logistic regression analysis confirmed that plateletocrit within $24 \mathrm{~h}$ after birth was the independent risk factor for preterm infants with hsPDA. Therefore, our findings demonstrated that the decrease in plateletocrit of the preterm infants was the independent risk factor for hsPDA

After the preterm infants are born, the strong contraction of arterial duct smooth muscle cannot often start, and the formed neointimal pad is sufficient to fill and block the residual cavity, making it difficult for the arterial duct to reshape and permanently close anatomically $[1,9]$. The aggregation and adhesion of the blood platelets inside the arterial duct have been suggested to be the influencing factors of arterial duct anatomical closures [3]. For example, in the transgenic mice with glycoprotein Ilb (blood platelet-specific integral protein) defect or platelet biosynthetic defect, the arterial duct remained open after $12 \mathrm{~h}$ of birth (the time for the mice arterial duct being spontaneously closed was 1-3 $\mathrm{h}$ after birth), as demonstrated by the angiography. Therefore, the poor blood platelet adhesion or aggregation function and reduced PLT will significantly affect the arterial duct closure $[3,10]$. Besides, a small single-center retrospective study of 123 preterm infants with the gestational age from 24 to 30 weeks in Germany demonstrated that the risk of PDA in the PLT $\leq 150 \times 10^{9} / \mathrm{L}$ group was increased by 13 times (OR, $13.1 ; 95 \% \mathrm{Cl}, 3.5-49.6)$ in comparison with the PLT $>150 \times 10^{9} / \mathrm{L}$ group, therefore, the thrombocytopenia was proposed to be an independent predicting factor for the arterial duct closure failure of the preterm infants [3]. It has also been shown that PLT $<100 \times 10^{9} / \mathrm{L}$ when birth was an independent risk factor for the occurrence of PDA on the preterm infants (OR, 4.50; 95\% Cl, 1.39-14.61) [4]. Consistent with previous reports, our study also showed that compared with the preterm infants with PLT $\geq 241.5 \times 10^{9} / \mathrm{L}, \geq 150 \times 10^{9} / \mathrm{L}$, and $\geq 100 \times 10^{9} / \mathrm{L}$, for the preterm infants with PLT $<241.5 \times 10^{9} / \mathrm{L},<150 \times$ $10^{9} / \mathrm{L}$, and $<100 \times 10^{9} / \mathrm{L}$ within $24 \mathrm{~h}$ after birth, the risk of hsPDA was increased by $1.876,2.169$, and 6.216 times. Further, a recent meta-analysis including 11 cohort studies and 3479 preterm infants with gestational age $<32$ weeks examined the relationship between the PLT and occurrence of PDA on the first day after birth and found that PDA was significantly correlated with PLT < $150 \times 10^{9} / \mathrm{L}(6$ studies, RR $1.215,95 \% \mathrm{Cl} 1.027-1.436)$ and PLT < 100 $\times 10^{9} / \mathrm{L}(5$ studies, RR $1.255,95 \% \mathrm{Cl} 1.034-1.525)$. The hsPDA was significantly correlated with PLT < $100 \times 10^{9} / \mathrm{L}$ (5 studies, RR $\left.1.254,95 \% \mathrm{Cl} 1.021-1.540\right)$, but the hsPDA was not significantly correlated with PLT $<150 \times 10^{9} / \mathrm{L}$ (6 studies, RR 1.289, 95\% Cl 0.925-1.795) [11].

There was also another point of view about PLT. PLT is lower than $150 \times 10^{9} / \mathrm{L}$ in hypoxia, preterm birth, and other pathological conditions, and these conditions can also lead to a higher incidence of PDA of the infants. Therefore, it has been speculated that the platelets in the circulation cannot help PDA closure and PLT also has no exact logic relationship with PDA closure, which is just a statistical correlation [9]. Recently, a large two-center retrospective cohort analysis was carried out on 1350 infants with very low birth weight in Germany, and the statistics showed that PLT within $24 \mathrm{~h}$ after birth had no relationship with the presence of PDA by the echocardiography examination within 4-5 days after birth [12]. The impaired platelet function rather than the reduction in PLT may have a certain relationship with the PDA. It has also been shown that there was no correlation between the closure of the arterial duct of the preterm infants and PLT [13]. The incidence of PDA in the PLT $<50 \times 10^{9} / \mathrm{L}$ group was similar to that in the PLT $>50 \times 10^{9} / \mathrm{L}$ group, and PDA incidence would be reduced only when the PLT was over $230 \times 10^{9} / \mathrm{L}$ [9]. Thus, with regard to the relationship between the thrombocytopenia and the incidence of PDA, there was still a large dispute. The reasons for the great difference among these research findings were still unclear, which could not be explained by the possible existence of difference in the neonatal intensive care unit (NICU) management [12]. It was recommended that a prospective study should be carried out to solve these disputes [9].

Alyamac et al. [14] retrospectively analyzed that the preterm infants with the median gestational age of 28 weeks and the median birth weight of $1060 \mathrm{~g}$ within 3 days after birth. Their results showed that, in the hsPDA group $(n=154)$, PLT was significantly lower than that in the $n P D A$ group $(n=207)$; PLT < $150 \times 10^{9} / L$ $(\mathrm{OR}=2.13,95 \% \mathrm{Cl}: 1.26-3.61)$ and PDW $>17 \%(\mathrm{OR}=2.68,95 \% \mathrm{Cl}: 1.41-5.09)$ were the risk factor for the occurrence of hsPDA; the MPV was not related to hsPDA. However, the mean PDW values in the nPDA group, nhsPDA group, and hsPDA group of the present study were $11.23 \%, 11.10 \%$, and $11.26 \%$, respectively, and PDW was not correlated with hsPDA. 
The single factor correlation analysis in our study showed that plateletocrit was also closely related to the closure of the arterial duct: for the preterm infants with plateletocrit $<0.245 \%$ and $0.09 \%$ within $24 \mathrm{~h}$ after birth, the risks of hsPDA were 1.749 and 5.407 times of preterm infants with plateletocrit $\geq 0.245 \%$ and $\geq 0.09 \%$, respectively. By the comparison of the areas under the ROC curves for predicting hsPDA of the preterm infants, it was prompted that the predictive value of plateletocrit was slightly better than PLT. Further, the multi-factor logistic regression analysis showed that plateletocrit instead of PLT within $24 \mathrm{~h}$ after birth was the independent risk factor of the hsPDA of the preterm infants. Plateletocrit is the product of PLT and MPV. The PLT can only reflect the platelet

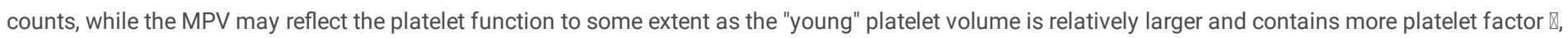
with the better adhesion and aggregation functions than the "elderly" platelets [1]. Therefore, the plateletocrit not only reflects the platelet counts but also the platelet function, rendering itself as an independent risk factor more directly related to the hsPDA of the preterm infants.

It is noteworthy that the area under the ROC curve for predicting the hSPDA of preterm infants by the plateletocrit within $24 \mathrm{~h}$ after birth was 0.748 , and the predictive value was merely moderate. The best prediction critical value was $0.245 \%$, and its sensitivity ( $68.2 \%$ ) and specificity (74.2\%) were not high due to the actions of multiple factors on the hsPDA in the preterm infants. In addition to the arterial duct maturity and genetic background, other factors affecting the arterial duct include the factors promoting the arterial duct smooth muscle contraction (oxygen, endothelin, calcium ion channel, catecholamines, Rho-kinase) and the factors inhibiting the arterial duct smooth muscle contraction (intraluminal pressure, prostaglandin, nitric oxide, carbon monoxide, potassium channel, cAMP, cGMP) [1, 2]. Among them, the vasodilator prostaglandins (especially prostaglandin E2) were the most important factors [1, 2] recognized currently in inhibiting arterial contraction in the late pregnancy. Therefore, although the low plateletocrit is the independent risk factor for the hsPDA of the preterm infants, further studies are required to elucidate other relevant risk factors. Similar to PLT, it also cannot be excluded that the plateletocrit level has merely a statistical correlation with PDA closure.

In summary, our study showed that the decrease in plateletocrit of the preterm infants within $24 \mathrm{~h}$ after birth was an independent risk factor of hsPDA on the $4^{\text {th }}-7^{\text {th }}$ day after birth. Combined with previous reports, it was speculated that the poor platelet function rather than simple reduction of the count may affect the closure of the arterial duct on the preterm infants, providing a theoretical basis for driving the arterial duct closure by the infusion of platelet-rich plasma [15].

\section{Conclusions}

The decrease in plateletocrit of the preterm infants within $24 \mathrm{~h}$ of birth was the independent risk factor of hsPDA on the 4 th-7th day of birth.

\section{Abbreviations}

patent ductus arteriosus (hsPDA)

platelet count (PLT)

receiver operating characteristic $(\mathrm{ROC})$

patent ductus arteriosus (PDA)

hemodynamically significant patent ductus arteriosus (hsPDA)

non-hemodynamically significant patent ductus arteriosus (nhsPDA)

Diagnosis criteria for non-patent ductus arteriosus (nPDA)

platelet distribution width (PDW)

mean platelet volume (MPV)

platelet-large cell ratio (P-LCR)

\section{Declarations}

\section{Ethics approval and consent to participate}

All parents, guardians or next of kin provided written informed consent for the minors to participate in this study.All procedures performed in this study were in accordance with the ethical standards of the institutional and/or national research committee and with the 1964 Helsinki declaration and its later amendments or comparable ethical standards.

\section{Consent for publication}

Written informed consent was obtained from all participants.

\section{Availability of data and materials}

The analyzed data sets generated during the study are available from the corresponding author on reasonable request.

Funding 
Jiangsu youth medical talents(QNRC2016385); Jiangsu maternal and child health research project(Neonatology F201645); Scientific research project of xuzho बKC16SL097, KC18034ه

\section{Competing Interests}

The authors declare that they had no competing interests.

\section{Authors' contributions}

YR designed the study, performed the study, collected the data, analyzed the data, and drafted the manuscript. XYG conceived of the study, performed the study, collected the data, analyzed the data, and helped to draft the manuscript. HYW participated in designing the study, performed the study, collected the data, analyzed the data, and revised the manuscript. BY, BJ, HLL, DDZ, and DH performed the study and collected the data. All authors read and approved the final manuscript.

\section{Acknowledgements}

Not applicable.

\section{References}

1. Gleason, C., Devaskar SU Avery's diseases of the newborn. 9 th ed. 2012, Elsevier Saunders. p. 751-761.

2. Benitz, W.E., Patent ductus arteriosus in preterm infants. Pediatrics, 2016. 137(1): p. e20153730.

3. Echtler, K., et al., Platelets contribute to postnatal occlusion of the ductus arteriosus. Nature medicine, 2010. 16(1): p. 75.

4. Dani, C., C. Poggi, and G. Fontanelli, Relationship between platelet count and volume and spontaneous and pharmacological closure of ductus arteriosus in preterm infants. American journal of perinatology, 2013. 30(05): p. 359-364.

5. El Hajjar, M., et al., Severity of the ductal shunt: a comparison of different markers. Archives of Disease in Childhood-Fetal and Neonatal Edition, 2005. 90(5): p. F419-F422.

6. Gleason, C., Devaskar SU Avery's diseases of the newborn. 9 th ed. 2012, Elsevier Saunders. p. 1056-1079.

7. Bas-Suárez, M.P., et al., Platelet counts in the first seven days of life and patent ductus arteriosus in preterm very low-birth-weight infants. Neonatology, 2014. 106(3): p. 188-194.

8. Wiedmeier, S.E., et al., Platelet reference ranges for neonates, defined using data from over 47000 patients in a multihospital healthcare system. Journal of perinatology, 2009. 29(2): p. 130.

9. Shah, N.A., et al., Relationship between circulating platelet counts and ductus arteriosus patency after indomethacin treatment. The Journal of pediatrics, 2011. 158(6): p. 919-923. e2.

10. Sallmon, H., et al., Ductal closure in neonates: a developmental perspective on platelet-endothelial interactions. Blood Coagulation \& Fibrinolysis, 2011. 22(3): p. 242-244.

11. Simon, S.R., et al., Platelet counts and patent ductus arteriosus in preterm infants: a systematic review and meta-analysis. Neonatology, 2015. 108(2): $\mathrm{p}$. 143-151.

12. Sallmon, H., et al., Thrombocytopenia in the first 24 hours after birth and incidence of patent ductus arteriosus. Pediatrics, 2012. 130(3): p. e623-e630.

13. Fujioka, K., et al., Does thrombocytopenia contribute to patent ductus arteriosus? Nature medicine, 2011. 17(1): p. 29.

14. Dizdar, E.A., et al., Low platelet count is associated with ductus arteriosus patency in preterm newborns. Early human development, 2012. 88(10): p. 813816.

15. Engür, M.A. and D. Engür, Platelet-rich plasma for patent ductus arteriosus: an orthopaedic surgeon's perspective. Cardiology in the Young, 2014. 24(3): p. 385-387.

\section{Tables}

Table 1. Demographic characteristics grouped according to the preterm infants' artery ducts 


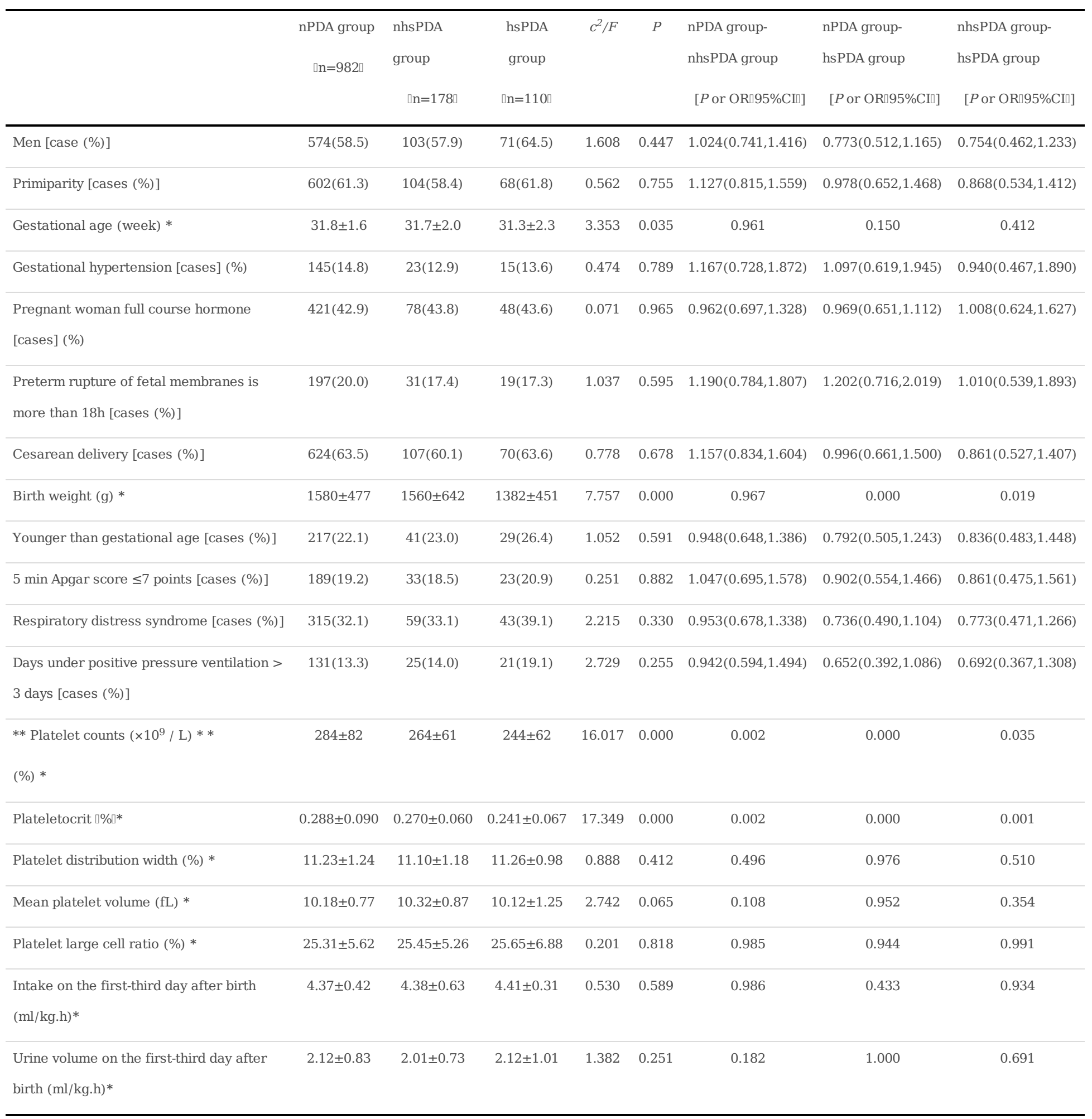

hsPDA: hemodynamically significant patent ductus arteriosus

nhsPDA: non-hemodynamically significant patent ductus arteriosus

nPDA: non-patent ductus arteriosus

CI: confidence interval

* The homogeneity of variance was not satisfied $(P<0.05)$ and the multiple-comparison was carried out with Tamhane's T2 method.

** The homogeneity of variance was satisfied $(P=0.09)$ and the multiple-comparison was carried out with LSD method. 
Table 2. The parameters of the ROC curve for predicting the hsPDA of preterm infants by four indicators

\begin{tabular}{|c|c|c|c|c|c|c|c|}
\hline Test variable & Area under the curve & $95 \%$ confidence interval & Standard error & $P$ & Best critical value & Sensitivity & Specificity \\
\hline Gestational age & 0.528 & $0.463-0.593$ & 0.033 & 0.332 & - & - & - \\
\hline Birth weight & 0.578 & $0.520-0.635$ & 0.029 & 0.007 & - & - & - \\
\hline Platelet counts & 0.703 & $0.655-0.751$ & 0.024 & 0.000 & $241.5 \times 10^{9} / \mathrm{L}$ & 0.782 & 0.670 \\
\hline Plateletocrit & 0.748 & $0.699-0.797$ & 0.025 & 0.000 & $0.245 \%$ & 0.682 & 0.742 \\
\hline
\end{tabular}

Table 3. The single factor correlation analysis of blood platelet parameters within $24 \mathrm{~h}$ after birth with the hsPDA of the preterm infants

\begin{tabular}{|c|c|c|c|c|}
\hline & & hsPDA [cases(\%)] & OR & $95 \%$ confidence interval \\
\hline \multirow[t]{6}{*}{ Platelet counts } & $<241.5 \times 10^{9} / \mathrm{L}$ & $56 / 469 \square 11.9 \% \square$ & 1.876 & $1.267-2.778$ \\
\hline & $\geq 241.5 \times 10^{9} / \mathrm{L}$ & $54 / 80106.7 \% \square$ & & \\
\hline & $<150 \times 10^{9} / \mathrm{L}$ & $12 / 74 \square 16.2 \% \square$ & 2.169 & $1.130-4.161$ \\
\hline & $\geq 150 \times 10^{9} / \mathrm{L}$ & 98/1196ロ8.2\%ロ & & \\
\hline & $<100 \times 10^{9} / \mathrm{L}$ & 4/11凸36.4\%॰* & 6.216 & $1.791-21.575^{*}$ \\
\hline & $\geq 100 \times 10^{9} / \mathrm{L}$ & $106 / 1259 \square 8.4 \% \square$ & & \\
\hline \multirow[t]{4}{*}{ Plateletocrit } & $<0.245 \%$ & $45 / 374 \square 12.3 \% \square$ & 1.749 & $1.171-2.611$ \\
\hline & $\geq 0.245 \%$ & 65/896ロ7.3\%ロ & & \\
\hline & $<0.09 \%$ & 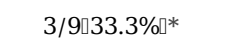 & 5.407 & $1.333-21.924^{*}$ \\
\hline & $\geq 0.09 \%$ & 107/1261₫8.5\%ロ & & \\
\hline
\end{tabular}

Table 4. Logistic regression analysis on the relevant factors of the hsPDA in the preterm infants

\begin{tabular}{|c|c|c|c|c|c|c|}
\hline Independent Variable & Classification assignment & Coefficient of regression & Standard error & Wals value & $P$ & OR (95\% confidence interval) \\
\hline Birth weight & $\begin{array}{l}500-1000 \mathrm{~g}=4,1000-1499 \mathrm{~g}=3 \\
1500-1999 \mathrm{~g}=2,2000-2499 \mathrm{~g}=1 \\
\geq 2500 \mathrm{~g}=0\end{array}$ & 0.265 & 0.109 & 5.891 & 0.015 & $1.303(1.052-1.613)$ \\
\hline Plateletocrit & $\begin{array}{l}<0.10 \%=3,0.10 \%-0.19 \%=2 \\
0.20 \%-0.29 \%=1, \geq 0.30 \%=0\end{array}$ & 1.202 & 0.155 & 60.013 & 0.000 & $3.328(2.455-4.512)$ \\
\hline Constant & & -4.149 & 0.303 & 187.306 & 0.000 & \\
\hline
\end{tabular}




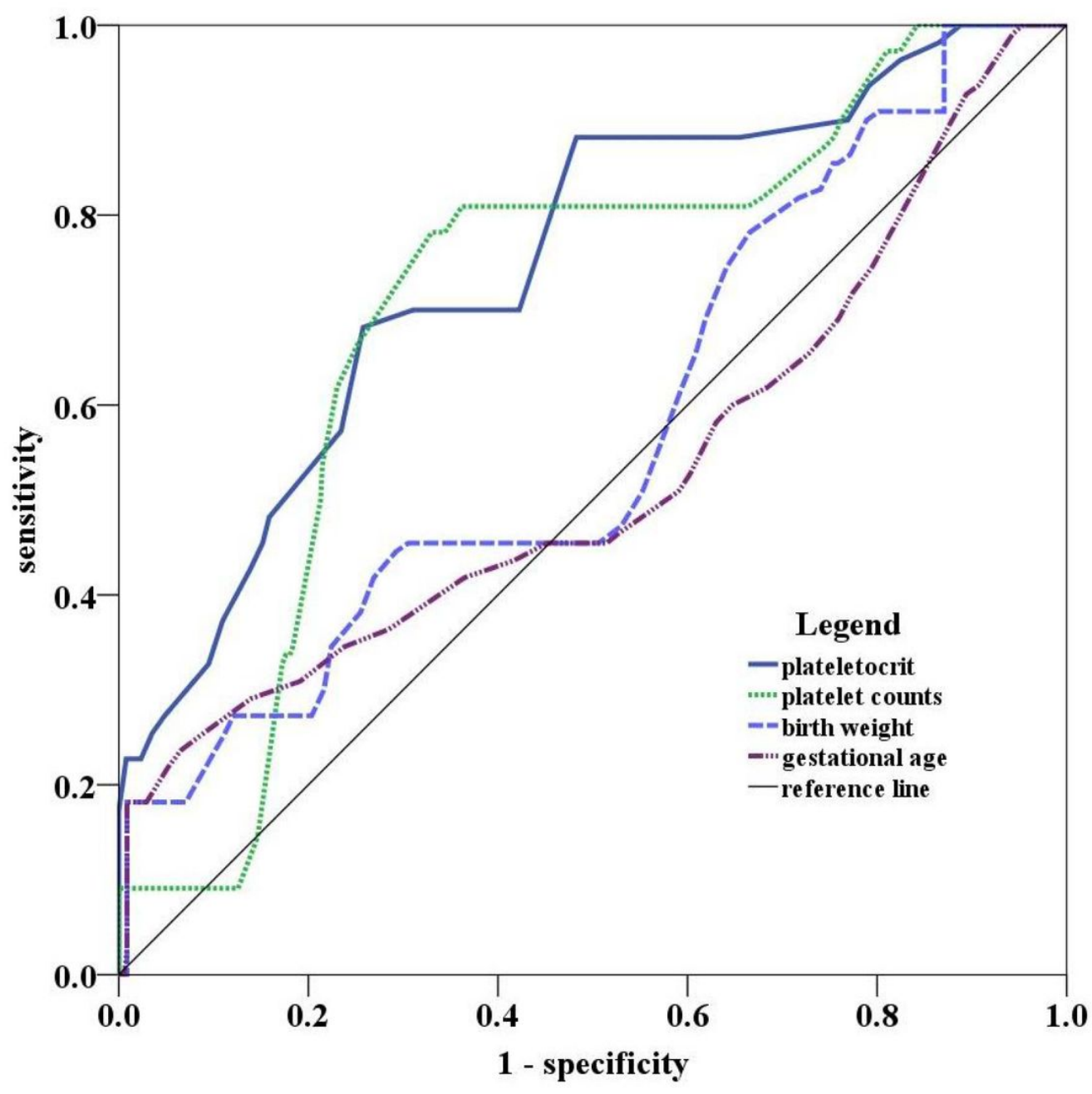

Figure 1

ROC curve for predicting the hsPDA of preterm infants by four indicators 